\title{
HAUSP as a therapeutic target for hematopoietic tumors (Review)
}

\author{
KANG WOO CHEON and KWANG-HYUN BAEK \\ Graduate School of Life Science and Biotechnology, Cell and Gene Therapy Research Institute, \\ Pochon CHA University, CHA General Hospital, Seoul 135-081, Korea
}

Received November 21, 2005; Accepted January 9, 2006

\begin{abstract}
. p53, one of the most important tumor suppressor proteins, plays an essential role in regulating the cell cycle and apoptosis by sensing the integrity of genome. Therefore, the level of p53 protein is critical for normal cellular homeostasis, and is known to be subtly regulated by ubiquitination and deubiquitination systems. Numerous genetic alterations of p53 have been reported in all types of tumors. In hematopoietic tumors, the mutations of $p 53$ gene are rare compared with solid tumors, which showed more than $50 \%$ frequency for $p 53$ mutations. According to this characteristic feature of hematological tumors, the therapeutic strategy for targeting the level of p53 may be valuable in anti-cancer treatment of hematological tumors. Herein, we deal with the posttranslational regulation of $\mathrm{p} 53$ via its specific ubiquitinating enzymes (Mdm2, Mdmx, COP1, Pirh2, ARF-BP1/Mule, and CHIP) and a deubiquitinating enzyme, herpesvirusassociated ubiquitin-specific protease (HAUSP). In this article, we review the regulatory mechanism of p53 via ubiquitination and deubiquitination system and suggest the several possible therapeutic strategies of targeting HAUSP, a deubiquitinating enzyme for $\mathrm{p} 53$, for treating hematopoietic tumors.
\end{abstract}

\section{Contents}

1. The mechanism of the ubiquitination/deubiquitination system

2. The role of p53 in hematopoietic tumors

3. Regulation of $\mathrm{p} 53$ by ubiquitination and deubiquitination

4. HAUSP as an evolutionarily conserved protein in mammals

5. Therapeutic approaches for targeting HAUSP

Correspondence to: Dr Kwang-Hyun Baek, Cell and Gene Therapy Research Institute, Pochon CHA University, CHA General Hospital, 605 Yeoksam 1-Dong, Kangnam-Gu, Seoul 135-081, Korea

E-mail: baek@cha.ac.kr

Key words: deubiquitinatating enzyme, HAUSP, hematopoietic tumors, p53, proteasome, ubiquitination

\section{The mechanism of the ubiquitination/deubiquitination system}

In view of cell biology, the regulation of selective proteolysis is largely mediated by the ubiquitin and proteasome system. In eukaryotes, most proteins, including transcription factors, cell-cycle regulators, signal transducers, and misfolded proteins, are processed and degraded by this mechanism $(1,2)$. Therefore, the regulation of the ubiquitination system plays a crucial role in pathological cellular physiology $(3,4)$. This regulated proteolysis occurs in the $26 \mathrm{~S}$ proteasome, which is composed by the association of the $20 \mathrm{~S}$ proteasome with two particles of the PA700 proteasome activator $(5,6)$. For $26 \mathrm{~S}$ proteasomal proteolysis, polyubiquitination of target proteins is necessary (Fig. 1).

Ubiquitin is a well-conserved 76-amino acid protein and is covalently attached to the amino group of internal Lys residue of target proteins by an enzymatic cascade of ubiquitinactivating enzymes (E1), ubiquitin-conjugating enzymes (E2), ubiquitin ligases (E3), and additional novel ubiquitination factors (E4), if necessary. In general, polyubiquitination is mediated by E1, E2, and E3 enzymes. However, recent reports demonstrated that the polyubiquitination of certain proteins essentially requires an additional ubiquitination factor, E4 $(1,7,8)$. For example, the parkin E3 ligase can monoubiquitinate Pael-receptor and further polyubiquitination requires the U-box-type E4 enzyme, CHIP (the C-terminus of Hsc70interacting protein), which was originally known as an E3 ligase for unfolded CFTR and glucocorticoid receptor (9). Another example of human E4 ligase is p300 (non-U-boxtype E4), which is known to have several biological activities, including transcriptional cofactor and histone acetyltransferase $(10,11)$. Following the monoubiquitination of target proteins, the second ubiquitin is attached to the Lys6, Lys11, Lys29, Lys48, or Lys63 amino acid residue of the first ubiquitin (12). This reaction can produce variable lengths of polyubiquitin chains, and the linkage type along with the length of chain may determine the fate of the target protein (13). Monoubiquitination and Lys63-linked polyubiquitination seem to have non-proteolytic roles, whereas Lys48-linked polyubiquitination plays a role in proteasomal degradation of target proteins (14).

The conjugation of ubiquitin to target proteins is reversible and the removal of ubiquitins is catalyzed by the deubiquitinating enzymes. Deubiquitinating enzymes hydrolyze polyubiquitin chains, resulting in rescue of target proteins from proteasomal degradation and release of free ubiquitins (15). In this ubiquitin-dependent proteolysis system, specific 


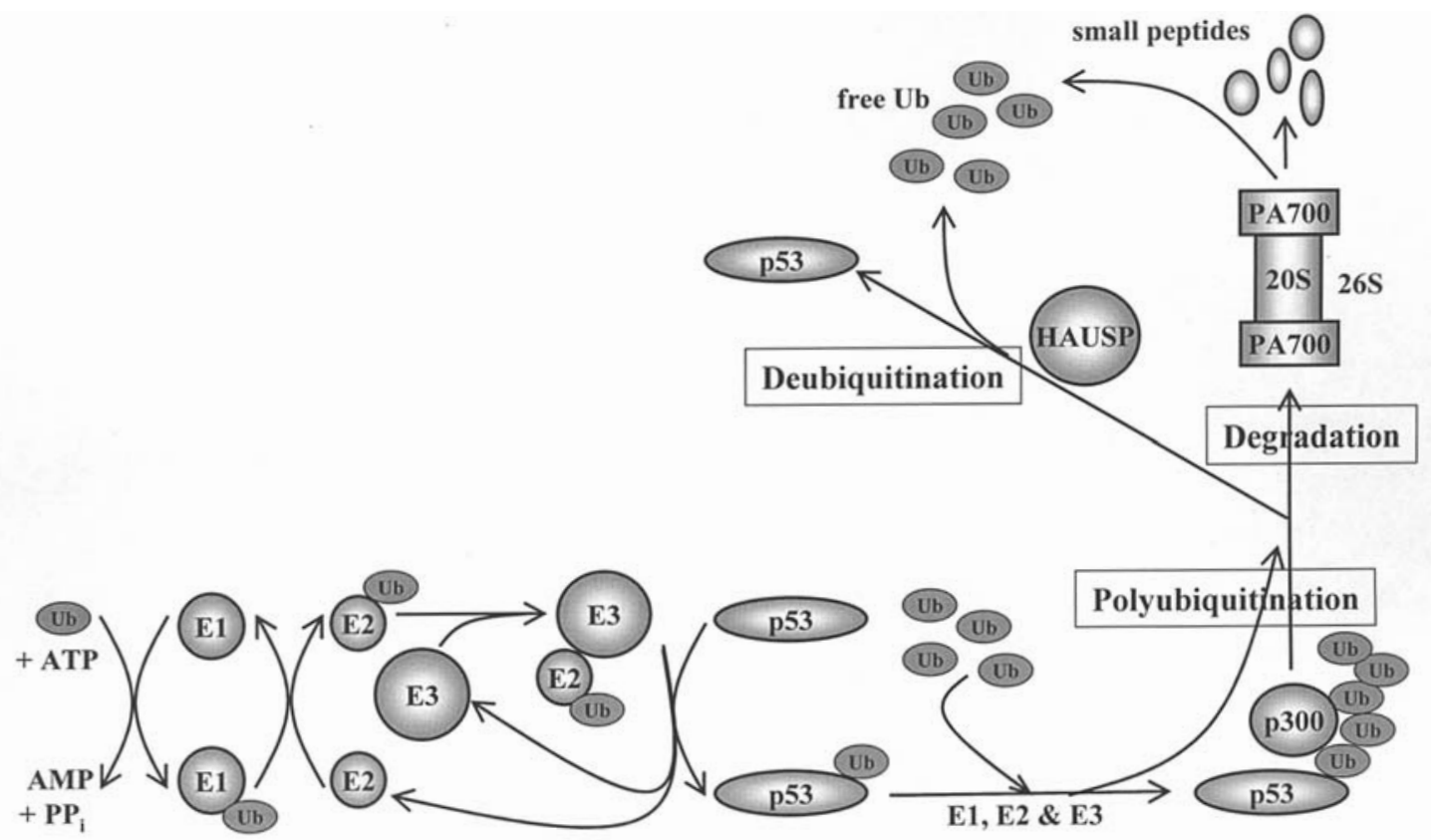

Figure 1. Schematic overview of the ubiquitination and deubiquitination system for p53. Monoubiquitination of p53 is mediated by E1, E2, and E3 enzymes with ubiquitin (Ub) and ATP. p300, an E4 enzyme, is known to be involved in polyubiquitination and the polyubiquitinated p53 is degraded by the $26 \mathrm{~S}$ proteasome. When the Mdm2 E3 ligase is highly expressed, p53 is polyubiquitinated by Mdm2 without p300. When a deubiquitinating enzyme, HAUSP, rescues the polyubiquitinated p53 before degradation, p53 recovers its protein functions, and free ubiquitins are recycled. Mdm2, COP1, Pirh2, Mule, and CHIP are known to be $\mathrm{E} 3$ ligases for $\mathrm{p} 53$.

recognition of substrate is necessary to prevent indiscriminate removal of essential proteins. To our knowledge, this specificity is determined by the interactions among substrates and their respective E2, E3, E4, and deubiquitinating enzyme by specific protein-protein interactions $(8,16)$. However, the precise mechanisms for these interactions are still undiscovered. The PEST motif has been known as a common feature of proteins that become ubiquitinated. This conserved hydrophilic motif is enriched in proline $(\mathrm{P})$, glutamine $(\mathrm{E})$, serine $(\mathrm{S})$, and threonine $(\mathrm{T})$ and is involved in the ubiquitination for rapid degradation of proteins (17). In addition, some of the substrates have to be post-translationally modified before they are recognized for ubiquitination. These post-translational modifications of substrates are commonly achieved by phosphorylation/dephosphorylation $(16,18)$, hydroxylation (19), or displacement of masking factors (20).

\section{The role of p53 in hematopoietic tumors}

p53 is well known as a tumor suppressor which leads to cell cycle arrest, cell senescence or apoptosis upon stress signals including DNA damage and oncogenic conditions (21). For example, $\gamma$-irradiation activates the ATM (ataxia-telangiectasia) kinase and the CHK-2 kinase, both of which can phosphorylate p53. Phosphorylated p53 acts as a transcription factor for inducing the $\mathrm{p} 21^{\mathrm{KIP}}$ family, which binds to the cyclin $\mathrm{D}$ and $\mathrm{E}$ complex and inhibits the release of E2Fs from the Rb:E2Fs complex (22). In addition, phosphorylated p53 induces the transcription of apoptosis-related genes, including Bax, NoxA, and Fas, which have the p53 binding site in their regulatory regions (23).

More than $50 \%$ of solid tumors have loss of wild-type $p 53$ expression because of deletions or point mutations $(24,25)$. A previous report showed that out of 118 lung tumors, 57 (48.3\%) had single (53 tumors) or double mutations (4 tumors) (25). Of these 57 tumors with $p 53$ mutations, 36 had missense mutations and 21 had null mutations that caused an aberrant-sized p53 protein (26). In contrast, hematopoietic tumors are less likely to have mutant $p 53$ in spite of their pathological diversity. In version R5 of the database for p53 mutations at the International Agency for Research on Cancer, only 14\% of 672 hematopoietic tumors have $p 53$ mutations (27). A recent metaanalysis demonstrated that only $11.9 \%$ of 4388 hematological tumors showed p53 mutations (Table I). In addition, 1482 tumors of acute myeloid leukemia (AML) and myelodysplastic syndrome (MDS) showed only $10 \%$ of p53 mutations. Chronic phase chronic myeloid leukemia (CP-CML) and Hodgkin's disease (HD) showed no p53 mutation (124 and 13 tumors, respectively). 690 tumors of acute lymphoblastic leukemia (ALL) showed 5\% of p53 mutations and multiple myeloma (MM) showed $3 \%$ (1 of 37 tumors) of p53 mutation. In contrast to the $p 53$ mutations, the altered level of $p 53$ expression was more frequent in hematopoietic tumors. Decreased expression of p53 was $30.4 \%$ of 23 hematological tumors (AML/MDS). Moreover, hematopoietic tumors with $R b$ point mutations or deletions were found in only $7.7 \%$ of 388 hematological malignancies, but $27.8 \%$ of 777 tumors have decreased expression of $\mathrm{Rb}$ (22). Collectively, these reports suggest that the majority of hematopoietic tumors may have wild-type p53 and its downstream of signal transduction pathway.

\section{Regulation of p53 by ubiquitination and deubiquitination}

p53 is a short-lived protein and its amount is maintained at a low level in normal cellular conditions due to its effect on cell cycle arrest and apoptosis. One mechanism of regulating 
Table I. A meta-analysis of alterations for $p 53$ and $R b$ genes in hematological tumors.

\begin{tabular}{llcc}
\hline Gene & \multicolumn{1}{c}{ Type of alteration } & $\begin{array}{c}\text { Number of } \\
\text { samples }\end{array}$ & $\begin{array}{c}\text { Incidence } \\
(\%)\end{array}$ \\
\hline$p 53$ & Mutation & $524 / 4388$ & 11.9 \\
& Deletion or rearrangement & $194 / 1378$ & 14.1 \\
& Decreased expression & $7 / 23$ & 30.4 \\
$R b$ & Mutation & $6 / 118$ & 5.1 \\
& Deletion or rearrangement & $24 / 270$ & 8.9 \\
& Decreased expression & $216 / 777$ & 27.8 \\
\hline
\end{tabular}

p53 is post-translational modification, including ubiquitination, phosphorylation, and acetylation (28). Key molecules involved in the process of p53 ubiquitination are E3 ligase Mdm2, and Mdmx (Fig. 2). It has been reported that Mdm2 has capacity for polyubiquitination and degradation of p53 when Mdm2 is highly expressed (29). Interestingly, p300 can also recognize monoubiquitinated p53 for ubiquitin chain elongation (11). It has been suggested that the Mdm2-mediated monoubiquitination of p53 results in nuclear export of p53, whereas polyubiquitination of p53 is crucial for degradation by the $26 \mathrm{~S}$ proteasome (30). Another important regulator of p53 function is Mdmx, a structural homologue of Mdm2 without E3 activity. Mdmx binds p53 and inhibits transcriptional activity by $\mathrm{p} 53$. Because Mdmx is a substrate for Mdm2-dependent ubiquitination, it can stabilize Mdm2 by the inhibition of selfubiquitination. Therefore, in normal cells, Mdmx plays a role in stabilizing Mdm2 and inhibiting p53 activity, which regulates normal cellular growth against apoptosis (31). In addition to Mdm2, additional E3 ligases for p53, Pirh2, COP1, ARF-BP1/ Mule, and CHIP, have been identified (32-35). ARF-BP1/Mule also has E3 ligase activity for Mcl-1 (36) and CHIP has previously known to possess both E3 activity for CFTR and glucocorticoid receptor and E4 activity for Pael-receptor (9). However, these additional E3 ligases for p53 have not been well studied yet. The precise cellular roles and regulation of COP1, Pirh2, ARF-BP1/Mule, and CHIP are not elucidated with regard to their cell- or tissue-specificity, and developmental stages. Therefore, the further investigation for synergistic interaction effects of these E3 ligases along with $\mathrm{Mdm} 2$ is required to disclose the precise regulatory mechanism of p53 ubiquitination.

p53 is ubiquitinated by Mdm2, resulting in the basal amount of p53 protein for preventing uncontrolled apoptosis. In normal status, Mdm2 binds to the transactivation domain of $\mathrm{p} 53$ to prevent its interaction with transcriptional machinery, and ubiquitinates $\mathrm{p} 53$ for degradation by the $26 \mathrm{~S}$ proteasome (37).

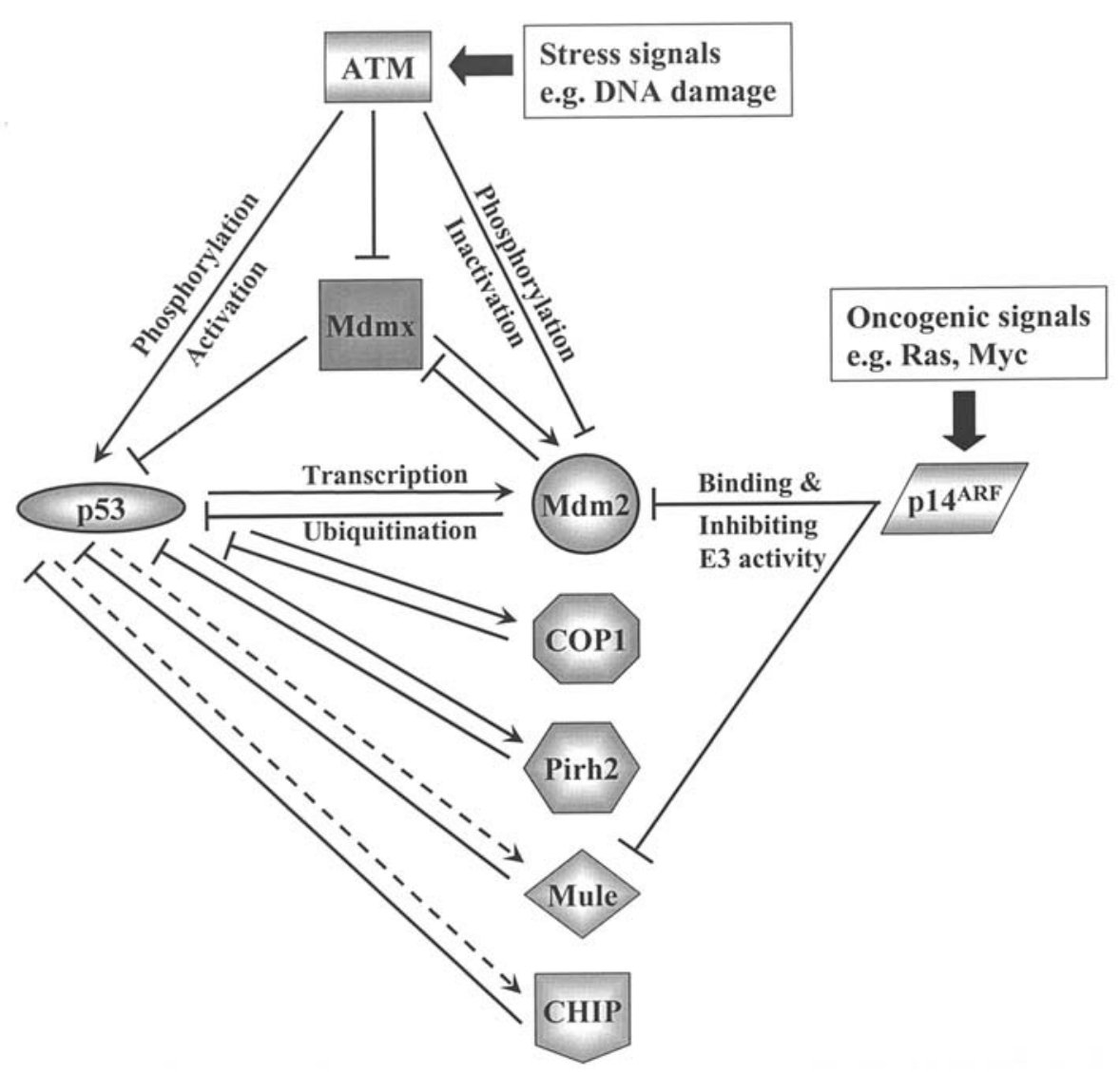

Figure 2. The regulatory model of p53 by the ubiquitination system. Phosphorylated Mdm2 inhibits the ubiquitination of p53. Phosphorylated p53 is activated as a transcription factor and induces the transcription of p53-specific E3 enzymes including Mdm2, COP1 and Pirh2, resulting in a negative feedback loop but this transcriptional regulation by Mule and CHIP (dots) has not been determined yet. Mdmx binds p53 and inhibits the transcriptional activity of p53. Mdmx also stabilizes Mdm2 by the inhibition of self-ubiquitination, and the ATM-dependent phosphorylation of Mdmx plays a crucial role in the ubiquitination and degradation of Mdmx by Mdm2. Arrows denote stimulatory effects, whereas horizontal bars indicate inhibitory influences. 


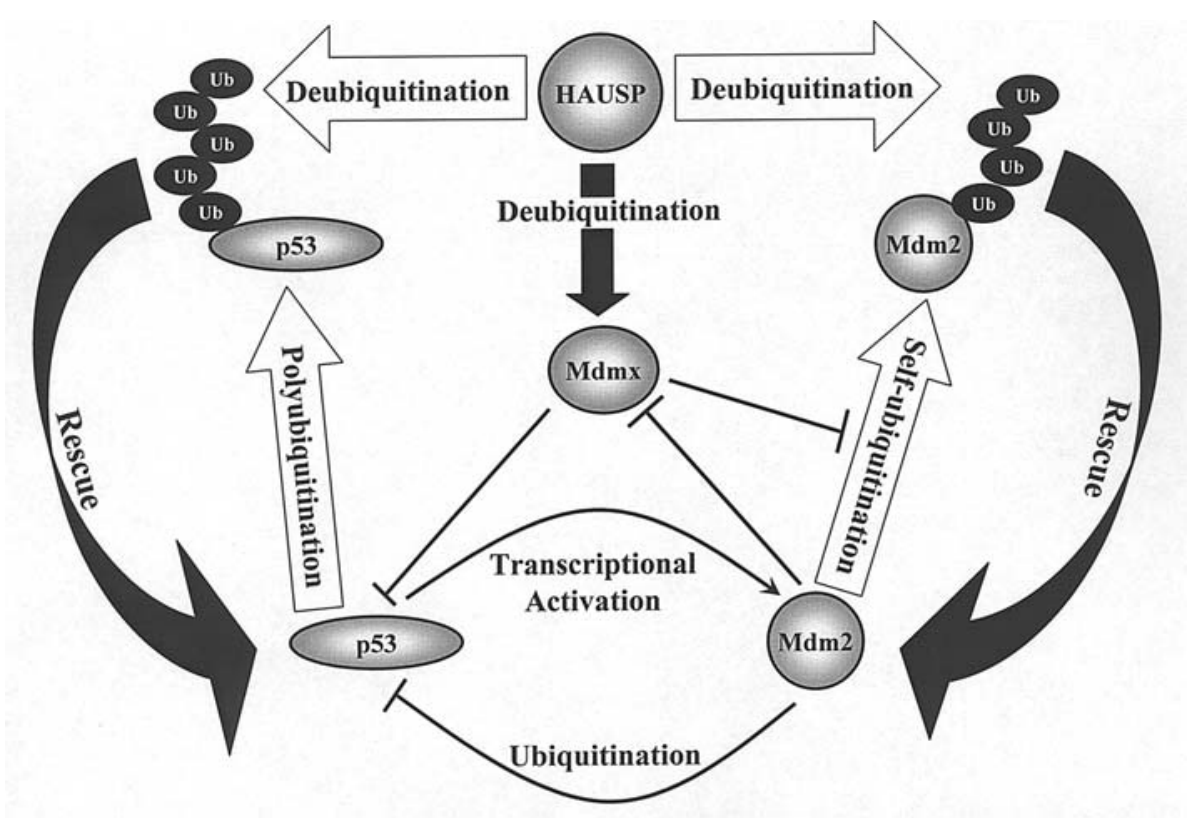

Figure 3. A regulatory model controlling the stability of p53 and Mdm2 by HAUSP. HAUSP can deubiquitinate p53, Mdm2, and Mdmx. The selfubiquitination activity of $\mathrm{Mdm} 2$ is important to regulate both $\mathrm{Mdm} 2$ and $\mathrm{p} 53$ at opposite levels. Mdmx stabilizes Mdm2 by inhibiting self-ubiquitination. HAUSP plays a crucial role for regulating the levels of p53, Mdm2, and Mdmx.

Consequently, the activity of p53 is maintained at a very low level in normal cells. In addition, activated p53 serves as a transcription factor for the $M d m 2$ gene, and up-regulation of Mdm2 eventually decreases the level of p53 by a negative feedback loop (38). Under stress conditions, such as DNA damage, the function of $\mathrm{Mdm} 2$ is inhibited by p14 ${ }^{\mathrm{ARF}}$ and ATM. p14 ${ }^{\mathrm{ARF}}$ is induced by oncoproteins (e.g. Ras and Myc) and binds both Mdm2 and ARF-BP1/Mule, resulting in inhibition of E3 ligase activity $(34,39)$. ATM is activated by stress signals and phosphorylates several target proteins, including p53, Mdm2 and Mdmx. ATM directly and indirectly induces the phosphorylation of Mdm2 and Mdmx, leading to decreased activity and stability of these proteins (40). Phosphorylated p53 is resistant to ubiquitination mediated by $\mathrm{Mdm} 2$, and the phosphorylation of Mdm2 by ATM inhibits the ubiquitination of p53. In addition, ATM-dependent phosphorylation of Mdmx plays a role in regulation of ubiquitination and degradation mediated by Mdm2 (41-43) (Fig. 2).

Deubiquitination is mediated by cysteine protease activity of deubiquitinating enzymes which possess highly conserved domains of the catalytic Cys, His, and Asp residues (6). HAUSP, one of the deubiquitinating enzymes, binds and stabilizes p53 by deubiquitination. HAUSP-overexpressed human lung carcinoma cells (H460) showed stabilization of p53 against polyubiquitination, resulting in p53-dependent cell cycle arrest and apoptosis (44). We also found the growth retardation and apoptosis of HAUSP-overexpressed cervical adenocarcinoma cells (HeLa) by transfection with a mouse orthologue of HAUSP (mHAUSP) $(45,46)$. Interestingly, disruption of HAUSP in human colorectal cancer (HCT116) cells and human osteosarcoma (U2OS) cells also showed the same results as shown in HAUSP-overexpressed cancer cells $(47,48)$. It is interesting that HAUSP can also deubiquitinate Mdm2 and Mdmx, resulting in stabilization of these proteins $(31,40)$. Therefore, it is possible that the disruption of HAUSP makes Mdm2 extremely unstable and fails to ubiquitinate p53, because Mdm2 is constitutively self-ubiquitinated and degraded (Fig. 3). When HAUSP is overexpressed, both p53 and $\mathrm{Mdm} 2$ are deubiquitinated and rescued, resulting in the increasing level of these proteins. In this situation, both selfubiquitination of Mdm2 and HAUSP-dependent rescue of p53 may lead to dominant function of p53 in the balance of p53 and Mdm2. When HAUSP is disrupted, both p53 and $\mathrm{Mdm} 2$ failed to be deubiquitinated. In this case, the absence of HAUSP-mediated Mdm2 deubiquitination and selfubiquitination of $\mathrm{Mdm} 2$ have a synergistic effect on the degradation of Mdm2. Consequently, ubiquitination of p53 is suppressed by an extremely low level of Mdm2, and p53 becomes stable and functional $(37,45,47,48)$.

\section{HAUSP as an evolutionarily conserved protein in mammals}

Human HAUSP (hHAUSP, also known as USP7) was originally identified by its binding activity for two herpesviral proteins, ICP0 (infected cell protein 0) and EBNA1 (Epstein-Barr nuclear antigen 1) (49,50). hHAUSP consists of 1102 amino acids and its molecular weight is approximately $135 \mathrm{kDa}$. Recently, we isolated the orthologues of hHAUSP in mouse (mHAUSP) and rat (rHAUSP) $(45,51)$. The open reading frame of both mHAUSP and rHAUSP consists of $3312 \mathrm{bp}$ and encodes a predicted protein of 1103 amino acids with a molecular weight of approximately $135 \mathrm{kDa}$. HAUSP is a well-conserved protein in mammals. hHAUSP shows 98.6\% amino acid identity with both mHAUSP and rHAUSP. Also, the amino acid identity is $99.6 \%$ between rHAUSP and mHAUSP (51). Using a partial proteolysis of HAUSP with MALDI-TOF/MS, four structural domains were found (52). Of these, three functional domains important for protein-protein interaction and enzymatic activity have been identified. The 


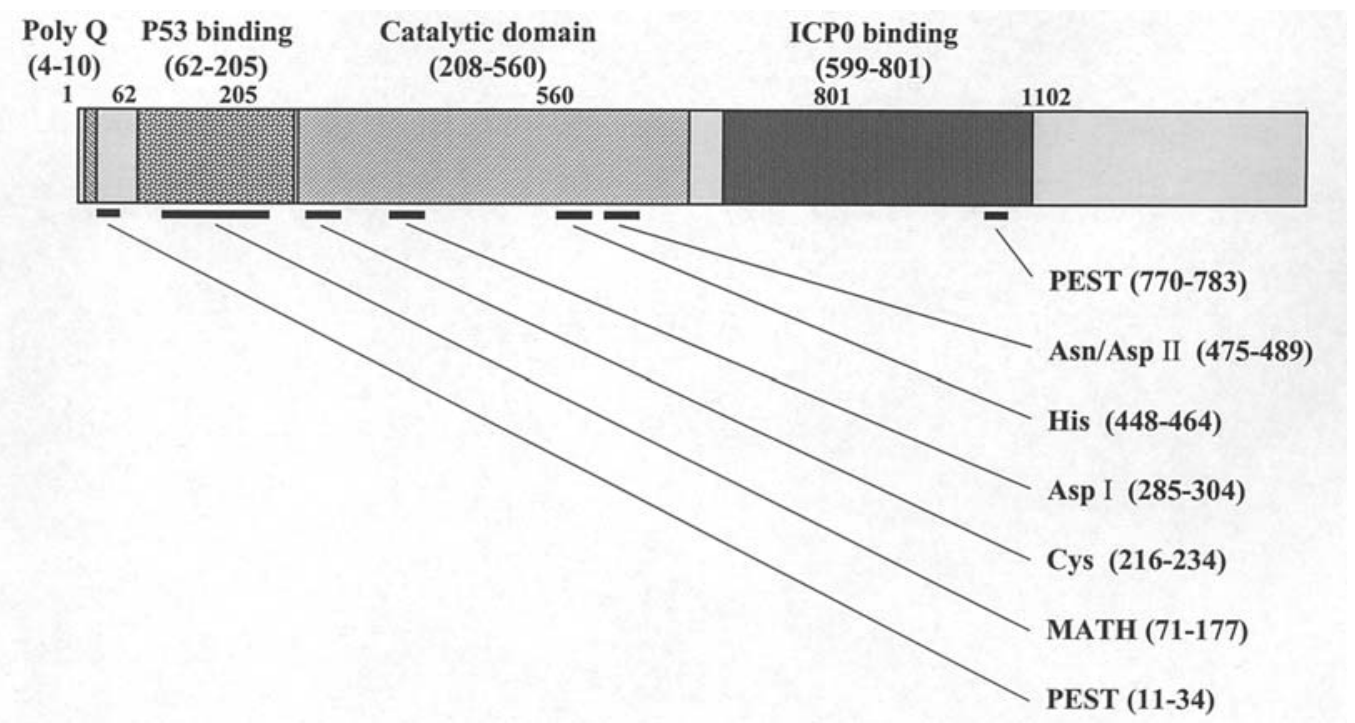

Figure 4. Schematic structure of the HAUSP protein. The domains of human HAUSP (PEST, MATH and catalytic domains) are illustrated with boxes and bold lines. The numbers of amino acid residues are in parentheses.

N-terminal domain (residues 62-205) is responsible for binding both EBNA1 and p53. Moreover, this domain includes the MATH (meprine and TRAF homology) domain, which is known to be critical for protein-protein interaction (45). The catalytic domain consists of approximately 350 amino acids (residues 208-560) and conserved three-domain architectures (Fingers, Palm, and Thumb) were found by crystal structure analysis (53). The catalytic domain has highly conserved Cys, Asp (I), His, and Asn/Asp (II) domains, which are one of the characteristic features of the deubiquitinating enzyme family and the amino acid sequences of these conserved domains are entirely identical in mouse, rat, and human (51). The domains of Cys, His, and Asn/Asp (II) are involved in the catalytic cleft and the domain of Asp (I) is located in the Thumb domain (53). ICP0 binding domain is located in the C-terminal 200 amino acids (residues 599-801) (52). The remaining C-terminal domain of 300 amino acids (residues 802-1102) has not been characterized. In addition, the domain responsible for interacting with $\mathrm{Mdm} 2$ has not been identified yet (Fig. 4). We recently found that rHAUSP is dimerized (51). However, the domain responsible for dimerization has not been identified yet and whether the dimerization of HAUSP is essential for its enzymatic activity remains to be investigated.

One of the interesting features of HAUSP is the N-terminal polyglutamine (poly $\mathrm{Q}$ ) region which is conserved among mouse, rat, and human. hHAUSP contains 7 Glu residues (residues 4-10) and 8 Glu residues (residues 4-11) in both mHAUSP and rHAUSP, respectively (51). Abnormal expansion of CAG repeat encodes poly $\mathrm{Q}$ tract. This mutational expansion of the polyglutamine tract in certain proteins results in neuro-degenerative diseases, including spinocerebellar ataxia 1,2, 3, 6, 7, and 17, Huntington's disease, spinobulbar muscular atrophy and dentatorubralpallidoluysian atrophy (54). Therefore, it remains to be elucidated whether the mutant HAUSP with the expanding poly Q tract exists and results in neurodegenerative diseases.

Interestingly, two conserved hydrophilic PEST motifs were found in the HAUSP proteins in human, mouse and rat using the PESTfind algorithm (http://www.at.ambnet.org/
embnet/tools/bio/PESTfind). One is located just after the poly $\mathrm{Q}$ tract in the N-terminus (24 amino acids: 11-34; KAGEQQLSEPEDMEMEAGDTDDPP) and the other is located in the C-terminus (14 amino acids: 770-783; KDDPE NDNSELPTA). It has been suggested that the PEST motif is involved in ubiquitination for the rapid degradation of proteins (17). Even though it is not known whether the ubiquitination of target proteins is directly mediated by the PEST motif, USP36 and HAUSP containing the PEST motif have been recently identified to be ubiquitinated $(51,55)$.

\section{Therapeutic approaches for targeting HAUSP}

As mentioned above, hematopoietic tumors are less likely to have mutations of $p 53$ and $R b$. This characteristic feature of hematopoietic tumors makes the hypothesis possible that the induction of p53 pathway via up- or down-regulation of HAUSP can force certain hematopoietic tumors into apoptosis as well as cell cycle arrest. HAUSP-targeted therapy is based on the proposition that the endogenous level of p53 expression (both transcription and translation) is not altered but the posttranslational regulation of $\mathrm{p} 53$ protein can be achieved by targeting HAUSP. We suggest that the amount of $\mathrm{p} 53$ protein has to be maintained within a normal range to prevent unwanted cell proliferation or growth retardation with the balance between p53 and its regulatory proteins, including Mdm2, Mdmx and HAUSP. According to this regulation, the strategy for targeting HAUSP is a valuable method for regulating the balance among these proteins. This therapeutic possibility regulating the endogenous level of p53 was already proven in non-hematopoietic tumor cells in vitro (44-48). A possible therapeutic strategy for targeting HAUSP includes the knock-down of HAUSP via siRNA, gene therapy for increasing expression of HAUSP, or delivery of HAUSP proteins into the cells. For example, HAUSP-CS, a catalytic mutant of HAUSP, in which a highly conserved Cys residue at the catalytic Cys domain was replaced by Ser (C224S in both mHAUSP and rHAUSP, C223S in hHAUSP), retained its strong binding ability to $\mathrm{p} 53$, but is functionally defective 
in deubiquitinating p53 in vitro $(44,45,51)$. Also, this catalytic mutant hHAUSP failed to deubiquitinate Mdm2 (31). In our study performed with mHAUSP-transfected HeLa cells, D296A and H465Q mutations also showed no enzyme activity, as identical with D295A and H464Q mutations in hHAUSP $(45,46)$. There are two possible models of how mutant HAUSP plays an inhibitory role for the endogenous intact HAUSP. One possibility is that a mutant HAUSP without deubiquitinating enzyme activity competitively binds p53 and Mdm2, resulting in failure of both $\mathrm{p} 53$ and $\mathrm{Mdm} 2$ deubiquitination. The other possibility is that mutant HAUSP acts as a dominant negative form to block the intact HAUSP function. We recently found that exogenously transfected HAUSP proteins can dimerize with endogenous HAUSP proteins in HeLa cells (51). This finding is concordant with our previous observation that B-lymphocyte-specific murine DUB-1 is dimerized (Baek, unpublished data). Our two observations imply that the dimerization of HAUSP may be required for its enzymatic functions. Therefore, the introduction of mutant HAUSP into cells may cause the formation of an inactive dimer complex between endogenous and mutant HAUSP enzymes.

We recently reported that deubiquitinating enzymes, USP36 and DUB-1A, are polyubiquitinated $(55,56)$. In addition, HAUSP is also polyubiquitinated, indicating the existence of HAUSP-specific E3 ligases and deubiquitinating enzymes (51). Therefore, investigation of HAUSP-specific E3 ligases and DUB enzymes may provide a novel strategy for targeting HAUSP and therapeutic tools for hematopoietic tumors. A recent report revealed that ICP0, a herpes simplex virus type 1 (HSV-1) E3 ligase, can ubiquitinate HAUSP, suggesting that HAUSP has the potential to be regulated by the ubiquitinproteasome pathway (57).

Finally, it has been suggested that HAUSP may contain several distinct binding sites, including p53, Mdm2, Mdmx, unidentified E3 and deubiquitinating enzymes for HAUSP, even though these binding regions and their specificity have not been investigated yet. This indicates that the highthroughput screening of chemical and peptide libraries for searching HAUSP binding molecules, which specifically interferes with binding of interacting proteins for HAUSP, may be valuable in finding binding site-specific drugs for HAUSP. The nutlin, as a small inhibitor molecule, is a good example of a p53 protein regulator. The nutlins bind Mdm2 in the p53 binding pocket with high selectivity and inhibit the p53-Mdm2 interaction, resulting in p53 stabilization (58).

For the application of HAUSP-targeting therapy in cancer treatment, its safety must be proven in normal cells, as with all types of anti-cancer therapy. Overexpression of HAUSP in mouse embryo fibroblasts (MEF) caused cell growth repression (44) and disruption of HAUSP in telomeraseimmortalized normal retinal-pigment epithelial cells (RPE) also arrested cell proliferation (48). In contrast to previous results, we observed that the overexpression of HAUSP did not affect the cell proliferation of normal fibroblasts (Baek, unpublished data). Therefore, further investigations and solutions for side effects of HAUSP-targeting therapies, including cell cycle arrest and possible apoptosis of normal cells, are preferentially required to provide a successful therapy for hematopoietic tumors.

\section{Acknowledgements}

The authors thank Drs Sook-Hwan Lee and Keun-Jai Yoo and the members of the Cell and Gene Therapy Research Institute at Pochon CHA University and CHA General Hospital for their critical comments on the manuscript. This study was supported by a grant (R01-2005-000-10292) from the interdisciplinary research program of the Korea Science and Engineering Foundation.

\section{References}

1. Hershko A and Ciechanover A: The ubiquitin system. Annu Rev Biochem 67: 425-479, 1998

2. Pickart CM: Mechanisms underlying ubiquitination. Annu Rev Biochem 70: 503-533, 2001

3. Wilkinson KD: Ubiquitin-dependent signaling: the role of ubiquitination in the response of cells to their environment. J Nutr 129: 1933-1936, 1999.

4. Wojcik C: Regulation of apoptosis by the ubiquitin and proteasome pathway. J Cell Mol Med 6: 25-48, 2002.

5. Rubin DM and Finley D: Proteolysis. The proteasome: a proteindegrading organelle? Curr Biol 5: 854-858, 1995.

6. Baek KH: Conjugation and deconjugation of ubiquitin regulating the destiny of proteins. Exp Mol Med 35: 1-7, 2003.

7. Ciechanover A: The ubiquitin-proteasome pathway: on protein death and cell life. EMBO J 17: 7151-7160, 1998.

8. Hoppe T: Multiubiquitination by E4 enzymes: 'one size' doesn;t fit all. Trends Biochem Sci 30: 183-187, 2005.

9. Imai Y, Soda M, Hatakeyama S, Akagi T, Hashikawa T, Nakayama KI and Takahashi R: CHIP is associated with Parkin, a gene responsible for familial Parkinson's disease, and enhances its ubiquitin ligase activity. Mol Cell 10: 55-67, 2002.

10. Scolnick DM, Chehab NH, Stavridi ES, Lien MC, Caruso L, Moran E, Berger SL and Halazonetis TD: CREB-binding protein and p300/CBP-associated factor are transcriptional coactivators of the p53 tumor suppressor protein. Cancer Res 57: 3693-3696, 1997.

11. Grossman SR, Deato ME, Brignone C, Chan HM, Kung AL, Tagami H, Nakatani Y and Livingston DM: Polyubiquitination of $\mathrm{p} 53$ by a ubiquitin ligase activity of p300. Science 300 : 342-344, 2003.

12. Pickart CM and Eddins MJ: Ubiquitin: structures, functions, mechanisms. Biochim Biophys Acta 1695: 55-72, 2004.

13. Hochstrasser M: Ubiquitination signaling: What's in a chain? Nat Cell Biol 6: 571-572, 2004.

14. Hicke L: Protein regulation by monoubiquitination. Nat Rev Mol Cell Biol 2: 195-201, 2001.

15. Wilkinson KD: Ubiquitination and deubiquitination: targeting of proteins for degradation by the proteasome. Semin Cell Dev Biol 11: 141-148, 2000.

16. Wood SA: Dubble or nothing? Is HAUSP deubiquitylating enzyme the final arbiter of p53 levels? Sci STKE 143: PE34, 2002.

17. Spencer ML, Theodosiou MT and Noonan DJ: NPDC-1, a novel regulator of neuronal proliferation, is degraded by the ubiquitin/ proteasome system through a PEST degradation motif. J Biol Chem 279: 37069-37078, 2004.

18. Chan EF, Gat U, McNiff JM and Fuchs E: A common human skin tumor is caused by activating mutations in B-catenin. Nat Genet 21: 410-413, 1999.

19. Ivan M, Kondo K, Yang H, Kim W, Valiando J, Ohh M, Salic A, Asara JM, Lane WS and Kaelin WG Jr: HIF $\alpha$ targeted for VHLmediated destruction by praline hydroxylation: implications for $\mathrm{O}_{2}$ sensing. Science 292: 464-468, 2001.

20. Salghetti SE, Muratani M, Wijnen H, Futcher B and Tansey WP: Functional overlap of sequences that activate transcription and signal ubiquitin-mediated proteolysis. Proc Natl Acad Sci USA 97: 3118-3123, 2000.

21. Jin S and Levine AJ: p53 functional circuit. J Cell Sci 114: 4139-4140, 2001

22. Krug U, Ganser A and Koeffler HP: Tumor suppressor genes in normal and malignant hematopoiesis. Oncogene 21: 3475-3495, 2002. 
23. Ko LJ and Prives C: p53: puzzle and paradigm. Genes Dev 10: 1054-1072, 1996.

24. Hainaut P, Hernandez T, Robinson A, Rodriguez-Tome P, Flores T, Hollstein M, Harris CC and Montesano R: IARC database of p53 gene mutations in human tumors and cell lines: updated compilation, revised formats and new visualisation tools. Nucleic Acids Res 26: 205-213, 1998.

25. Hollstein M, Sidransky D, Vogelstein B and Harris CC: p53 mutations in human cancers. Science 253: 49-53, 1991.

26. Mori S, Ito G, Usami N, Yoshioka H, Ueda Y, Kodama Y, Takahashi M, Fong KM, Shimokata K and Sekido Y: p53 apoptotic pathway molecules are frequently and simultaneously altered in nonsmall cell lung carcinoma. Cancer 100: 1673-1682, 2004.

27. Hernandez-Boussard T, Rodriguez-Tome P, Montesano R and Hainaut P: IARC p53 mutation database: a relational database to compile and analyze p53 mutations in human tumors and cell lines. International Agency for Research on Cancer. Hum Mutat 14: 1-8, 1999.

28. Brooks $\mathrm{CL}$ and $\mathrm{Gu} \mathrm{W}$ : Ubiquitination, phosphorylation and acetylation: the molecular basis for p53 regulation. Curr Opin Cell Biol 15: 164-171, 2003.

29. Li M, Brooks CL, Wu-Baer F, Chen D, Baer R and Gu W: Mono- versus polyubiquitination: differential control of $\mathrm{p} 53$ fate by Mdm2. Science 302: 1972-1975, 2003.

30. Brooks $\mathrm{CL}$ and $\mathrm{Gu} \mathrm{W}$ : Monoubiquitination: the signal for $\mathrm{p} 53$ nuclear export? Cell Cycle 3: 436-438, 2004

31. Meulmeester E, Maurice MM, Boutell C, Teunisse AF, Ovaa H, Abraham TE, Dirks RW and Jochemsen AG: Loss of HAUSPmediated deubiquitination contributes to DNA damage-induced destabilization of Hdmx and Hdm2. Mol Cell 18: 565-576, 2005.

32. Leng RP, Lin Y, Ma W, Wu H, Lemmers B, Chung S, Parant JM, Lozano G, Hakem R and Benchimol S: Pirh2, a p53-induced ubiquitin-protein ligase, promotes p53 degradation. Cell 112: 779-791, 2003.

33. Dornan D, Wertz I, Shimizu H, Arnott D, Frantz GD, Dowd P, O'Rourke K, Koeppen H and Dixit VM: The ubiquitin ligase COP1 is a critical negative regulator of p53. Nature 429: 86-92, 2004.

34. Chen D, Kon N, Li M, Zhang W, Qin J and Gu W: ARF-BP1/ Mule is a critical mediator of the ARF tumor suppressor. Cell 121: 1071-1083, 2005.

35. Esser C, Scheffner M and Hohfeld J: The chaperone-associated ubiquitin ligase CHIP is able to target p53 for proteasomal degradation. J Biol Chem 280: 27443-27448, 2005.

36. Zhong Q, Gao W, Du F and Wang X: Mule/ARF-BP1, a BH3only E3 ubiquitin ligase, catalyzes the polyubiquitination of Mcl-1 and regulates apoptosis. Cell 121: 1085-1095, 2005.

37. Michael D and Oren M: The p53-Mdm2 module and the ubiquitin system. Semin Cancer Biol 13: 49-58, 2003.

38. Wu L and Levine AJ: Differential regulation of the p21/WAF-1 and $\mathrm{mdm} 2$ genes after high-dose UV irradiation: p53-dependent and $\mathrm{p} 53$-independent regulation of the $\mathrm{mdm} 2$ gene. Mol Med 3: 441-451, 1997.

39. Honda R and Yasuda H: Association of p19 (ARF) with Mdm2 inhibits ubiquitin ligase activity of Mdm2 for tumor suppressor p53. EMBO J 18: 22-27, 1999.

40. Meulmeester E, Pereg Y, Shiloh Y and Jochemsen AG: ATMmediated phosphorylations inhibit Mdmx/Mdm2 stabilization by HAUSP in favor of p53 activation. Cell Cycle 4: 1166-1170, 2005.

41. Canman CE, Lim DS, Cimprich KA, Taya Y, Tamai K, Sakaguchi K, Appella E, Kastan MB and Siliciano JD Activation of the ATM kinase by ionizing radiation and phosphorylation of p53. Science 281: 1677-1679, 1998.
42. Shieh SY, Taya Y and Prives C: DNA damage-inducible phosphorylation of $\mathrm{p} 53$ at $\mathrm{N}$-terminal sites including a nove site, Ser20, requires tetramerization. EMBO J 18: 1815-1823, 1999

43. Chen L, Gilkes DM, Pan Y, Lane WS and Chen J: ATM and Chk2-dependent phosphorylation of Mdmx contribute to p53 activation after DNA damage. EMBO J 24: 3411-3422, 2005.

44. Li M, Chen D, Shiloh A, Luo J, Nikolaev AY, Qin J and Gu W: Deubiquitination of p53 by HAUSP is an important pathway for p53 stabilization. Nature 416: 648-653, 2002.

45. Lim SK, Shin JM, Kim YS and Baek KH: Identification and characterization of murine mHAUSP encoding a deubiquitinating enzyme that regulates the status of p53 ubiquitination. Int $\mathbf{J}$ Oncol 24: 357-364, 2004.

46. Yoo KJ, Lee HJ, Lee H, Lee KY, Lee SH, Chung HM and Baek KH: Expression and functional analyses of mHAUSP regulating apoptosis of cervical adenocarcinoma cells. Int $\mathbf{J}$ Oncol 27: 97-104, 2005.

47. Li M, Brooks CL, Kon N and Gu W: A dynamic role of HAUSP in the p53-Mdm2 pathway. Mol Cell 13: 879-886, 2004.

48. Cummins JM, Rago C, Kohli M, Kinzler KW, Lengauer C and Vogelstein B: Tumour suppression: disruption of HAUSP gene stabilizes p53. Nature 428: 486, 2004.

49. Meredith M, Orr A and Everett R: Herpes simplex virus type 1 immediate-early protein Vmw110 binds strongly and specifically to a 135-kDa cellular protein. Virology 200: 457-469, 1994.

50. Holowaty MN, Zeghouf M, Wu H, Tellam J, Athanasopoulos V, Greenblatt $\mathrm{J}$ and Frappier L: Protein profiling with Epstein-Barr nuclear antigen-1 reveals an interaction with the herpesvirusassociated ubiquitin-specific protease HAUSP/USP7. J Biol Chem 278: 29987-29994, 2003.

51. Lee HJ, Kim MS, Kim YK, Oh YK and Baek KH: HAUSP, a deubiquitinating enzyme for $\mathrm{p} 53$, is polyubiquitinated, polyneddylated, and dimerized. FEBS Lett 579: 4867-4872, 2005

52. Holowaty MN, Sheng Y, Nguyen T, Arrowsmith C and Frappier L: Protein interaction domains of the ubiquitin-specific protease, USP7/HAUSP. J Biol Chem 278: 47753-47761, 2003.

53. Hu M, Li P, Li M, Li W, Yao T, Wu JW, Gu W, Cohen RE and Shi Y: Crystal structure of a UBP-family deubiquitinating enzyme in isolation and in complex with ubiquitin aldehyde. Cell 111: 1041-1054, 2002.

54. Tarlac V and Storey E: Role of proteolysis in polyglutamine disorders. J Neurosci Res 74: 406-416, 2003.

55. Kim MS, Kim YK, Kim YS, Seong M, Choi JK and Baek KH Deubiquitinating enzyme USP36 contains the PEST motif and is polyubiquitinated. Biochem Biophys Res Commun 330: 797-804, 2005

56. Baek KH, Kim MS, Kim YS, Shin JM and Choi HK: DUB-1A, a novel deubiquitinating enzyme subfamily member, is polyubiquitinated and cytokine-inducible in B-lymphocytes. J Biol Chem 279: 2368-2376, 2004

57. Boutell C, Canning M, Orr A and Everett RD: Reciprocal activities between herpes simplex virus type 1 regulatory protein ICP0, a ubiquitin E3 ligase, and ubiquitin-specific protease USP7. J Virol 79: 12342-12354, 2005.

58. Thompson T, Tovar C, Yang H, Carvajal D, Vu BT, Xu O, Wahl GM, Heimbrook DC and Vassilev LT: Phosphorylation of p53 on key serines is dispensable for transcriptional activation and apoptosis. J Biol Chem 279: 53015-53022, 2004 